\title{
Nature or Nurture? Determining the Heritability of Human Striatal Dopamine Function: an [18F]-DOPA PET Study
}

\author{
Paul RA Stokes*, 1,2, Paul Shotbolt', Mitul A Mehta ${ }^{3,4}$, Eric Turkheimer ${ }^{5}$, Aaf Benecke ${ }^{2}$, Caroline Copeland', \\ Federico E Turkheimer ${ }^{4}$, Anne R Lingford-Hughes ${ }^{2}$ and Oliver D Howes' \\ 'Psychiatric Imaging Group, MRC Clinical Sciences Centre, Imperial College London, Hammersmith Hospital, London, UK; ${ }^{2}$ Centre for \\ Neuropsychopharmacology, Division of Brain Sciences, Department of Medicine, Imperial College London, London, UK; ${ }^{3}$ Centre for Neuroimaging \\ Sciences, Institute of Psychiatry at King's College London, London, UK; ${ }^{4}$ Division of Experimental Medicine, Department of Medicine, Imperial \\ College London, London, UK; ${ }^{5}$ Department of Psychology, University of Virginia, Charlottesville, VA, USA
}

Striatal dopamine function is important for normal personality, cognitive processes and behavior, and abnormalities are linked to a number of neuropsychiatric disorders. However, no studies have examined the relative influence of genetic inheritance and environmental factors in determining striatal dopamine function. Using [I 8F]-DOPA positron emission tomography (PET), we sought to determine the heritability of presynaptic striatal dopamine function by comparing variability in uptake values in same sex monozygotic (MZ) twins to dizygotic (DZ) twins. Nine MZ and 10 DZ twin pairs underwent high-resolution [I 8F]-DOPA PET to assess presynaptic striatal dopamine function. Uptake values for the overall striatum and functional striatal subdivisions were determined by a Patlak analysis using a cerebellar reference region. Heritability, shared environmental effects and non-shared individual-specific effects were estimated using a region of interest (ROI) analysis and a confirmatory parametric analysis. Overall striatal heritability estimates from the ROI and parametric analyses were 0.44 and 0.33 , respectively. We found a distinction between striatal heritability in the functional subdivisions, with the greatest heritability estimates occurring in the sensorimotor striatum and the greatest effect of individual-specific environmental factors in the limbic striatum. Our results indicate that variation in overall presynaptic striatal dopamine function is determined by a combination of genetic factors and individual-specific environmental factors, with familial environmental effects having no effect. These findings underline the importance of individual-specific environmental factors for striatal dopaminergic function, particularly in the limbic striatum, with implications for understanding neuropsychiatric disorders such as schizophrenia and addictions.

Neuropsychopharmacology (2013) 38, 485-49I; doi:I0.1038/npp.2012.207; published online 24 October 2012

Keywords: twin; PET; dopamine; heritability; [I8F]-DOPA; environment

\section{INTRODUCTION}

The striatal dopamine system plays an important role in both normal human behavior and neuropsychiatric disorders. In healthy populations, variation in markers of striatal dopaminergic function have been associated with differences in the expression of personality traits (Egerton et al, 2010b; Gjedde et al, 2010; Kim et al, 2011; Reeves et al, 2007), social status (Martinez et al, 2010), and cognitive function (Cervenka et al, 2008; Landau et al, 2009; Reeves et al, 2005). In psychiatric disorders, increased dopaminergic function is associated with highly heritable disorders such as schizophrenia (Howes and Kapur, 2009; Kegeles et al, 2010), and decreased function with stimulant (Martinez et al, 2007) and alcohol addiction (Volkow et al, 2007). Although

\footnotetext{
*Correspondence: Dr PRA Stokes, Psychiatric Imaging Group, MRC Clinical Sciences Centre, Imperial College, Hammersmith Hospital Campus, Du Cane Road, London, UK WI2 ONN, Tel: + 44(0)2075942679, Fax: + 44(0)2075946548, E-mail: paul.stokes@imperial.ac.uk Received 7 June 2012; revised 31 August 2012; accepted 17 September 2012
}

variation in striatal dopamine function occurs between individuals, little is known about the relative contribution of genetic inheritance and environmental influences on human striatal dopamine function. A better understanding of this is vital to the interpretation of the effects of variability in striatal dopaminergic function in both health and disease.

The human striatum can be functionally subdivided into three distinct areas: the limbic striatum, which mediates reward and motivation; the associative striatum, which mediates working memory and executive functions; and the sensorimotor striatum, which mediates movement (Cools and D'Esposito, 2011; Haber et al, 2000; Lappin et al, 2009). Variation in dopamine function in each striatal subdivision is associated with alterations in corresponding behavioral modalities (Cools et al, 2009; Dreher et al, 2009; Forbes et al, 2009). Presynaptic dopamine function in these subdivisions can be imaged in the living human brain using 6-[18F]-L-DOPA ([18F]-DOPA) positron emission tomography (PET). [18F]-DOPA PET provides a measure of aromatic acid decarboxylase activity, which converts DOPA to dopamine, and as such the accumulation of [18F]-DOPA within the brain reflects the functional 
integrity of presynaptic dopamine synthesis (Cumming et al, 1997). We have previously shown that [18F]-DOPA PET provides good reliability both for the overall striatum and for the striatal subdivisions (Egerton et al, 2010a).

The relative influence of genetic and environmental factors on human biological systems can be examined through the use of twin studies. Twin studies assume that monozygotic (MZ) twins are genetically identical, so that most variation between MZ twins is due to environmental influences, and that dizygotic (DZ) twins share on average $50 \%$ of their genetic material. A comparison of intrapair variation between $\mathrm{MZ}$ and $\mathrm{DZ}$ twin pairs allows for the estimation of heritability $\left(h^{2}\right)$, the proportion of variation attributable to the additive effects of genes. Twin studies also allow for the estimation of shared (familial) environmental variance $\left(c^{2}\right)$ and for the estimation of non-shared (individual-specific) environmental variance $\left(e^{2}\right)$. Although twin studies provide a powerful way of interrogating the effects of genetic and environmental factors on biological variance, there have so far been no twin studies of the human dopamine system.

In this study we used [18F]-DOPA PET in healthy same sex $\mathrm{MZ}$ and $\mathrm{DZ}$ twin pairs to determine the relative contribution of genetic and environmental factors to variation in human presynaptic striatal dopamine function. We hypothesized that presynaptic striatal dopamine function would be more strongly influenced by genetic inheritance than by environmental influences, given the association between human dopaminergic function and highly heritable disorders such as schizophrenia.

\section{MATERIALS AND METHODS}

\section{Participants}

Nine MZ same sex twin pairs (four male pairs, mean age $37.7 \pm 2.9$ years) and 10 DZ same sex twin pairs (five male pairs, mean age $39.5 \pm 3.2$ years) were recruited to the study by means of public advertisement. The DZ twin cohort group was also used as a normal comparator group for a recently published study of [18F]-DOPA uptake in twins discordant for schizophrenia (Shotbolt et al, 2011). None of the twins had a history of a significant mental health disorder, alcohol or drug dependency, serious physical illness, past neurological disorders or previous use of psychotropic medications. Six MZ twins and four DZ twins were current cigarette smokers at the time of imaging and four MZ twins and seven DZ twins had previously used cannabis recreationally. Four DZ twins (two twin pairs) had previously used cocaine (mean duration since last use: 12 months \pm 16.1 ); one twin had only ever used cocaine once, one used $\sim 12$ times over his lifetime, lifetime cocaine usage data are unavailable for the other two twins. All volunteers gave written informed consent for the study, which was approved by both the Hammersmith Research Ethics Committee and the Administration of Radioactive Substances Advisory Committee, UK.

\section{Zygosity Determination}

At the time of imaging, zygosity determination was based on resemblance from direct observation, as well as verbal statements from twin pairs and their relatives. Twin zygosity status was subsequently confirmed after the completion of imaging, once we had obtained consent specifically for this from volunteers, by both DNA analysis and the use of the 'peas in the pod' twin zygosity questionnaire. For the DNA analysis, a multiplex PCR technique was used to determine whether twins shared 16 highly polymorphic microsatellite markers (from 7 to 17 alleles in length). This analysis yields a $>99.99 \%$ accuracy rate for MZ twins and 100\% accuracy rate for DZ twins. The 'peas in the pod' twin zygosity questionnaire provides a $>96 \%$ accuracy rate for the determination of twin zygosity (Reed et al, 2005).

\section{PET Scanning and Image Acquisition}

Each twin underwent one [18F]-DOPA PET scan. Volunteers were asked to abstain from alcohol for $24 \mathrm{~h}$ before the scan and from smoking cigarettes for $4 \mathrm{~h}$ before the scan. Each volunteer underwent a urine drug screen analysis on the day of the scan for cannabis, cocaine, methamphetamine, amphetamine, opiates, and benzodiazepines. Any volunteer who produced a positive urine drug screen on the scan day was excluded from the study. All volunteers received $150 \mathrm{mg}$ carbidopa and $400 \mathrm{mg}$ entacapone orally $1 \mathrm{~h}$ before scanning to reduce the formation of radiolabeled metabolites (Wahl et al, 1994). PET scans were performed using an ECAT/EXACT3D PET scanner (Siemens/CTI, Knoxville, USA), with a spatial resolution of $4.8 \pm 0.2 \mathrm{~mm}$ and sensitivity of $69 \mathrm{cps} / \mathrm{Bq} / \mathrm{ml}$. A 5 -min transmission scan was carried out before radiotracer injection to correct for attenuation and scatter. Approximately $150 \mathrm{MBq}$ of 6-[18F]L-DOPA ([18F]-DOPA) was administered by bolus intravenous injection $30 \mathrm{~s}$ after the start of the emission scan that lasted $95 \mathrm{~min}$. Emission data were acquired in list mode and rebinned into 26 time frames. High-resolution brain images were reconstructed from 95 planes with a slice spacing of $2.42 \mathrm{~mm}$ using the $3 \mathrm{D}$ re-projection algorithm.

\section{Image Analysis}

All scans were first corrected for head movement using frame-by-frame (FBF) realignment as previously described (Montgomery et al, 2006). A region of interest (ROI) analysis was performed using an atlas comprised of the three functional subdivisions of the striatum; limbic, associative, and sensorimotor striatum along with the cerebellum. These functional striatal subdivisions are anatomically analogous to the ventral striatum (limbic striatum), precommissural dorsal putamen, precommissural dorsal caudate and post-commissural dorsal caudate (associative striatum), and post-commissural putamen (sensorimotor striatum) (Martinez et al, 2003). An [18F]DOPA template (McGowan et al, 2004) was spatially transformed to the individual PET space of each movement corrected PET summation image using statistical parametric mapping (SPM5; Wellcome Department of Cognitive Neurology, London, England) and the resulting deformation matrix was then applied to the atlas (Meyer et al, 1999). This procedure allows for ROI's to be placed automatically on individual [18F]-DOPA PET images without observer bias. A graphical Patlak analysis was used to calculate 
[18F]-DOPA influx constants (Ki values) for the whole striatal ROI and right and left functional striatal subdivisions relative to uptake in the cerebellar reference region (Egerton et al, 2010a; Hoshi et al, 1993; Patlak and Blasberg, 1985).

\section{Heritability Analysis}

[18F]-DOPA heritability values were calculated by fitting the data to a multilevel model with random effects. For observed variable $y$ for twin $i$ in family $f, y$ is expressed as a family mean $b_{\text {of }}$ and within family variation $\varepsilon_{\mathrm{if}}$ :

$$
y_{\mathrm{i} f}=b_{o f}+\varepsilon_{\mathrm{i} f}
$$

With $\varepsilon_{\text {if }} \sim N\left(0, \sigma_{w}^{2}\right)$.The family mean $b_{\text {of }}$ is expressed as a population mean $B_{0}$ and deviations of family means $\mu_{\mathrm{o} f}$ around the population mean.

$$
b_{\mathrm{of}}=B_{0}+\mu_{\mathrm{of}}
$$

with

$$
\mu_{\mathrm{of}} \sim N\left(0, \sigma_{B}^{2}\right) \text {. }
$$

The between and within family variances were reparameterized into useful terms for biometric analysis. First, the ratio of the between pair variance to the total phenotypic variation produces the intraclass correlation for family membership:

$$
r_{i c c}=\frac{\sigma_{B}^{2}}{\sigma_{B}^{2}+\sigma_{w}^{2}}
$$

The between and within variances, directly computed in $\mathrm{MZ}$ and DZ twins without use of biometric constraints, were reparameterized into the three biometric variance components A (additive genetic effects), C (shared familial environment effects), and $\mathrm{E}$ (non-shared individual-specific environment effects). In MZ twins:

$$
\begin{aligned}
& \sigma_{w}^{2}=E \\
& \sigma_{B}^{2}=A+C \\
& \text { Whereas in D } \\
& \sigma_{w}^{2}=\frac{1}{2} A+E \\
& \sigma_{B}^{2}=\frac{1}{2} A+C
\end{aligned}
$$

Whereas in DZ twins:

Finally, the ACE variance components were standardized into percentages of variation attributable to $h^{2}, c^{2}, e^{2}$, for example the heritability, $h^{2}$, which is equal to:

$$
\frac{A}{A+C+E}
$$

The models were fitted in Mplus using full information maximum likelihood estimation. Negative ACE variances were noted, fixed to zero and the model was then rerun to generate final results.

Additionally, we also applied an optimized voxel-level ICC analysis (for unordered pairs) to each set of twins (Caceres et al, 2009). This has the advantage that reliability of any given region can be calculated from the distribution of reliability from all voxels in that region, and also that reliability in a given region can be expressed relative to the whole brain.

\section{RESULTS}

\section{Zygosity Determination}

The zygosity status of 16/18 MZ twins (88\%) and 14/20 DZ $(70 \%)$ twins was confirmed using either DNA analysis results or questionnaire results. Of the remaining twins, one $\mathrm{MZ}$ pair declined and two $\mathrm{DZ}$ pairs and two individual $\mathrm{DZ}$ twins could not be re-contacted to obtain their consent for this.

\section{ROI Heritability Results}

MZ and DZ twin pair [18F]-DOPA uptake values for the overall and limbic striatum are shown in Figure 1 (corresponding figures for the associative and sensorimotor striatum are displayed in Supplementary Figures S1 and S2). Overall mean striatal [18F]-DOPA Ki values were 0.0146 (0.0009) for the MZ group and $0.0142(0.0014)$ for the DZ group. MZ and DZ ICC values along with estimated $h^{2}, c^{2}$, and $e^{2}$ values are shown in Table 1 . The heritability value for overall striatal [18F]-DOPA uptake was 0.44 . Heritability estimates were highest in the sensorimotor striatum (right: 0.51 , left: 0.64 ) and lowest in the limbic striatum (right: 0.21 , left: 0 ). The influence of non-shared individual-specific environmental factors on overall striatal uptake variability was 0.56. Individual-specific environmental effects showed the reverse pattern to heritability in the functional striatal subdivisions with the highest values in the limbic striatum (right: 0.53, left: 0.70), and the lowest values in the sensorimotor striatum (right: 0.49 , left: 0.36 ). The pattern of these results did not change with the removal of one MZ twin pair whose zygosity status could not be confirmed (see Supplementary Table 1) or with the exclusion of two twin pairs with previous recreational experience of cocaine use (see Supplementary Table 2).

\section{Parametric Heritability Results}

Overall striatal MZ and DZ ICC values from the parametric image analysis were 0.44 and 0.27 , respectively. These results generated $h^{2}, c^{2}$, and $e^{2}$ estimates for the overall striatum of $0.33,0.1$, and 0.56, respectively. Areas of high within-pair striatal correlations (parametric ICC values between 0.5 and 1) are displayed in Figure 2.

\section{DISCUSSION}

In this study we have determined, for the first time to our knowledge, the relative influence of genetic inheritance and environmental factors over presynaptic striatal dopamine function in the living human brain. Our main finding is that overall striatal presynaptic dopaminergic function is determined by a combination of genetic factors, which accounted for $44 \%$ of the variance from the ROI analysis and $33 \%$ from the parametric analysis, and individualspecific environmental factors that accounted for $56 \%$ of the variance from both the ROI and parametric analyses. We found that shared familial environmental factors had very 

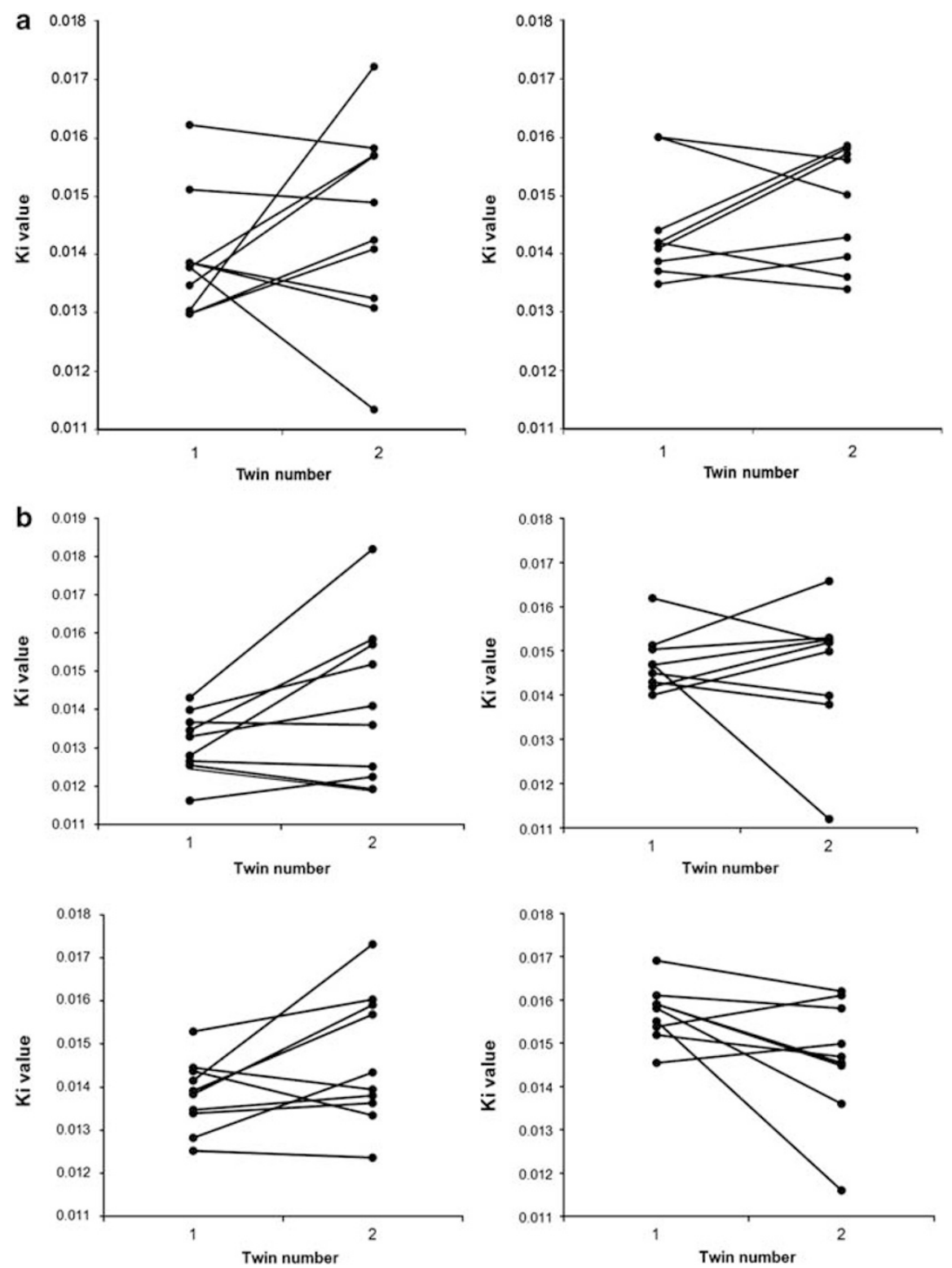

Figure I (a) Overall striatal [ I 8F]-DOPA Ki values for MZ (right) and DZ (left) twin pairs. (b) Right and left limbic striatal [I 8F]-DOPA Ki values for MZ (right) and DZ (left) twin pairs (right limbic: upper row, left limbic: lower row).

little influence over human presynaptic striatal dopamine function. Intriguingly, heritability estimates varied between functional striatal subdivisions with the highest heritability values found in the sensorimotor striatum and the lowest in the limbic striatum.

These results have a number of implications for the interpretation of variation in striatal dopaminergic function in both health and disease. Our finding that individual-specific environmental factors account for $56 \%$ of the variance of presynaptic striatal dopamine function is consistent with previous findings that striatal dopaminergic function is adaptive to environmental influences. For example in primates, striatal dopaminergic function can be altered by change in social hierarchy, and in humans striatal dopaminergic function is associated with social status and perceived social support (Martinez et al, 2010; Morgan et al, 2002). Interestingly, our results contrast to the heritability of the $5 \mathrm{HT}_{2 \mathrm{~A}}$ receptor, the only other neurochemical system where heritability has been established in vivo, in which the individual-specific environmental influences accounted for $<10 \%$ of the variance of the cortical signal (Pinborg et al, 2008). One explanation for this contrast could be due to differences in measurement error, which contributes to the individual environmental variance term, between the two tracers. However, as the test-retest variability of [18F]-DOPA uptake (4-6\%) (Egerton et al, 2010a) is similar to [18F]altanserin variability (5-12\%) (Haugbol et al, 2007), this does not account for the large differences in the individualspecific environmental variance observed between the two systems. There would therefore seem to be a marked difference in the adaptability of these two neurochemical systems to non-shared individual-specific environmental influences. 
Table I Heritability Estimates $\left(h^{2}\right)$ and Estimates of the Effect of Shared $\left(c^{2}\right)$ and Non-shared Environmental $\left(e^{2}\right)$ Factors on Presynaptic Striatal Dopaminergic Function ( ) =Standard Error

\begin{tabular}{lcccccc}
\hline Striatal area & MZ ICC & $\mathbf{D Z}$ ICC & $\mathbf{h}^{\mathbf{2}}$ & $\mathbf{c}^{\mathbf{2}}$ & $\mathbf{e}^{\mathbf{2}}$ \\
\hline Right limbic & 0.17 & 0.36 & $0.21(0.75)$ & $0.26(0.54)$ & $0.53(0.3 \mathrm{I})$ \\
Left limbic & 0.08 & 0.28 & 0 & $0.30(0.21)$ & $0.70(0.2 \mathrm{I})$ \\
Right associative & 0.36 & 0.13 & $0.46(0.33)$ & 0 & $0.54(0.33)$ \\
Left associative & 0.30 & 0.18 & $0.51(0.28)$ & 0 & $0.49(0.28)$ \\
Right sensorimotor & 0.64 & 0.02 & $0.51(0.22)$ & 0 & $0.49(0.22)$ \\
Left sensorimotor & 0.67 & 0.04 & $0.64(0.23)$ & 0 & $0.36(0.23)$ \\
Overall striatum & 0.39 & 0.06 & $0.44(0.36)$ & 0 & $0.56(0.36)$ \\
\hline
\end{tabular}

We interpret the relatively greater influence of individualspecific environmental factors on limbic presynaptic striatal dopamine function as a reflection of the greater responsiveness of the limbic striatum to environmental factors when compared with other functional striatal areas. A number of studies support this interpretation; the greatest increases in striatal dopamine release produced by environmental stimuli such as stress, monetary reward and playing a video game occurs in the ventral striatum rather than more dorsal striatal functional subdivisions (Koepp et al, 1998; Pappata et al, 2002; Pruessner et al, 2004; Schott et al, 2008). Use of stimulants, such as amphetamine, may also act preferentially to increase limbic striatal dopamine release. This is supported by amphetamine challenge studies in healthy volunteers that report greater dopamine release in the ventral striatum in healthy volunteers compared with other striatal subregions (Martinez et al, 2003; Willeit et al, 2008). Indeed in modeling sensitization to stimulants in humans, Boileau et al (2006) reported that sensitization of the striatal dopamine system is greatest in the ventral striatum and with repeated exposure progressively involves the dorsal caudate and putamen. This is exactly the pattern of sensitization we would predict based on our findings.

The influence of individual-specific environmental influences on presynaptic dopamine function also has implications for the interpretation of studies of dopaminergic function in neuropsychiatric conditions such as schizophrenia and addictions. We previously found no increases in [18F]-DOPA uptake in healthy DZ co-twins of patients with schizophrenia, at high genetic risk of developing the disorder, which supports the role that environmental factors may play in influencing increased [18F]-DOPA uptake found in schizophrenia (Shotbolt et al, 2011). The findings from this present study indicate that disease alterations in the limbic striatum in both schizophrenia and addictions are more likely to reflect individual-specific environmental than inherited risk factors for these conditions and we would suggest that future environmental risk studies focus particularly on limbic striatal function.

There are several limitations to the present study. First, the number of volunteers imaged was small in comparison to epidemiological twin studies, restricting our statistical power. However, we have previously shown very good testretest reliability (intraclass correlation coefficient $>0.84$ ) for striatal [18F]-DOPA PET imaging on the scanner used for this study with a smaller volunteer sample size (Egerton et al, 2010a). Furthermore, our cohort of volunteers is larger

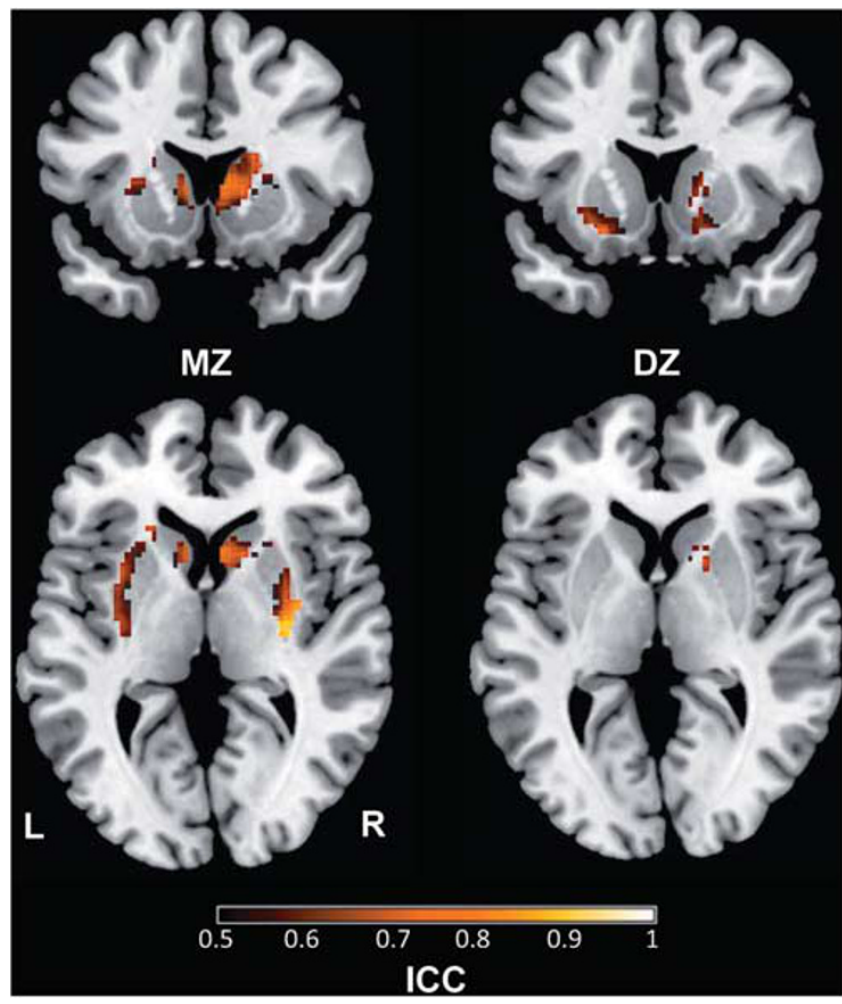

Figure 2 Areas of high within twin pair striatal [18F]-DOPA uptake correlations (parametric ICC values between 0.5 and I).

than the $5 \mathrm{HT}_{2 \mathrm{~A}}$ twin study (Pinborg et al, 2008). Second, the reliability of $[18 \mathrm{~F}]-\mathrm{DOPA}$ PET measurements varies between functional subdivisions studied, with the greatest variability occurring in the limbic striatum. Limbic heritability estimates may therefore be less reliable than other functional subdivisions that potentially could influence the heritability pattern between functional subdivisions. However, within subject variability in the limbic striatum, although greater than in sensorimotor striatum, is nevertheless low, $<8 \%$ (Egerton et al, 2010a), so this is unlikely to account for the $\sim 40 \%$ difference we found in heritability between the limbic and sensorimotor striatal regions. Third, the zygosity status of one pair of MZ twins was not confirmed by genetic analysis as they declined to provide consent for zygosity testing. Although the possibility remains that this pair may have been $\mathrm{DZ}$, this is unlikely given the physical similarity of the pair at the time of imaging and a previous study confirming zygosity status in over $95 \%$ of twins who reported that they were MZ (Reed et al, 2005). Moreover, the pattern of our results did not change with the exclusion of this pair from our analysis.

In summary, we have demonstrated that overall presynaptic dopamine function is determined by a combination of genetic and individual-specific environmental factors and that heritability varies between functional striatal subdivisions. These findings underline the adaptability of the striatal dopaminergic system, and particularly the limbic striatum, to individual-specific environmental influences. We would suggest that it is important to explore whether similar patterns of heritability also occur in twin studies of striatal dopamine release and post-synaptic striatal dopamine $\mathrm{D}_{2} / \mathrm{D}_{3}$ receptor availability. Our findings 
also reinforce the importance of identifying the underlying neurochemical mechanisms that mediate the effects of unique environmental factors on striatal dopamine function.

\section{ACKNOWLEDGEMENTS}

We acknowledge the support and guidance of Professor Paul Grasby in the design and implementation of this study. This study was funded by grant funding (Grant code: U.1200. 04.007.00001.01) from the Medical Research Council, UK.

\section{DISCLOSURE}

Dr Mehta received research grant support from Eli Lilly, acted as a consultant for Cambridge Cognition and UCB PLC, and received a speaker's fee from Shire Pharmaceuticals. Professor Eric Turkheimer was funded by NIH and was a paid consultant on a NIH funded twin study at the University of Washington. He received no consulting funding from any private organizations. Professor Lingford-Hughes has received honoraria from Janssen-Cilag, Pfizer, Servier, Lundbeck and from the British Association for Psychopharmacology. She has provided consultancy to NET Device Corp, received research funding from Archimedes, Lundbeck, Pfizer, and Schering, and holds research grants with GlaxoSmithKline. Dr Howes has received research funding from the MRC (Grant: MC-A656-5QD30) for this study. He has received investigator-led charitable research funding or has been on the speaker bureaux for Astra-Zeneca, BMS, Eli Lilly, and Jansenn Cilag.

\section{REFERENCES}

Boileau I, Dagher A, Leyton M, Gunn RN, Baker GB, Diksic M et al (2006). Modeling sensitization to stimulants in humans: an [11C]raclopride/positron emission tomography study in healthy men. Arch Gen Psychiatry 63: 1386-1395.

Caceres A, Hall DL, Zelaya FO, Williams SC, Mehta MA (2009). Measuring fMRI reliability with the intra-class correlation coefficient. Neuroimage 45: 758-768.

Cervenka S, Backman L, Cselenyi Z, Halldin C, Farde L (2008). Associations between dopamine D2-receptor binding and cognitive performance indicate functional compartmentalization of the human striatum. Neuroimage 40: 1287-1295.

Cools R, D'Esposito M (2011). Inverted-U-shaped dopamine actions on human working memory and cognitive control. Biol Psychiatry 69: e113-e125.

Cools R, Frank MJ, Gibbs SE, Miyakawa A, Jagust W, D’Esposito M (2009). Striatal dopamine predicts outcome-specific reversal learning and its sensitivity to dopaminergic drug administration. J Neurosci 29: 1538-1543.

Cumming P, Deep P, Rousset O, Evans A, Gjedde A (1997). On the rate of decarboxylation of dopa to dopamine in living mammalian brain. Ann NY Acad Sci 835: 274-308.

Dreher JC, Kohn P, Kolachana B, Weinberger DR, Berman KF (2009). Variation in dopamine genes influences responsivity of the human reward system. Proc Natl Acad Sci USA 106: 617-622.

Egerton A, Demjaha A, McGuire P, Mehta MA, Howes OD (2010a). The test-retest reliability of 18F-DOPA PET in assessing striatal and extrastriatal presynaptic dopaminergic function. Neuroimage 50: 524-531.

Egerton A, Rees E, Bose SK, Lappin JM, Stokes PR, Turkheimer FE et al (2010b). Truth, lies or self-deception? Striatal $\mathrm{D}(2 / 3)$ receptor availability predicts individual differences in social conformity. Neuroimage 53: 777-781.

Forbes EE, Brown SM, Kimak M, Ferrell RE, Manuck SB, Hariri AR (2009). Genetic variation in components of dopamine neurotransmission impacts ventral striatal reactivity associated with impulsivity. Mol Psychiatry 14: 60-70.

Gjedde A, Kumakura Y, Cumming P, Linnet J, Moller A (2010). Inverted-U-shaped correlation between dopamine receptor availability in striatum and sensation seeking. Proc Natl Acad Sci USA 107: 3870-3875.

Haber SN, Fudge JL, McFarland NR (2000). Striatonigrostriatal pathways in primates form an ascending spiral from the shell to the dorsolateral striatum. J Neurosci 20: 2369-2382.

Haugbol S, Pinborg LH, Arfan HM, Frokjaer VM, Madsen J, Dyrby TB et al (2007). Reproducibility of 5-HT2A receptor measurements and sample size estimations with [18F]altanserin PET using a bolus/infusion approach. Eur J Nucl Med Mol Imaging 34: 910-915.

Hoshi H, Kuwabara H, Leger G, Cumming P, Guttman M, Gjedde A (1993). 6-[18F]fluoro-L-dopa metabolism in living human brain: a comparison of six analytical methods. J Cereb Blood Flow Metab 13: 57-69.

Howes OD, Kapur S (2009). The dopamine hypothesis of schizophrenia: version III-the final common pathway. Schizophr Bull 35: 549-562.

Kegeles LS, Abi-Dargham A, Frankle WG, Gil R, Cooper TB, Slifstein $\mathrm{M}$ et al (2010). Increased synaptic dopamine function in associative regions of the striatum in schizophrenia. Arch Gen Psychiatry 67: 231-239.

Kim JH, Son YD, Kim HK, Lee SY, Cho SE, Kim YB et al (2011). Association of harm avoidance with dopamine D2/3 receptor availability in striatal subdivisions: a high resolution PET study. Biol Psychol 87: 164-167.

Koepp MJ, Gunn RN, Lawrence AD, Cunningham VJ, Dagher A, Jones $\mathrm{T}$ et al (1998). Evidence for striatal dopamine release during a video game. Nature 393: 266-268.

Landau SM, Lal R, O’Neil JP, Baker S, Jagust WJ (2009). Striatal dopamine and working memory. Cereb Cortex 19: 445-454.

Lappin JM, Reeves SJ, Mehta MA, Egerton A, Coulson M, Grasby PM (2009). Dopamine release in the human striatum: motor and cognitive tasks revisited. J Cereb Blood Flow Metab 29: $554-564$.

Martinez D, Narendran R, Foltin RW, Slifstein M, Hwang DR, Broft A et al (2007). Amphetamine-induced dopamine release: markedly blunted in cocaine dependence and predictive of the choice to self-administer cocaine. Am J Psychiatry 164: 622-629.

Martinez D, Orlowska D, Narendran R, Slifstein M, Liu F, Kumar D et al (2010). Dopamine type $2 / 3$ receptor availability in the striatum and social status in human volunteers. Biol Psychiatry 67: 275-278.

Martinez D, Slifstein M, Broft A, Mawlawi O, Hwang DR, Huang Y et al (2003). Imaging human mesolimbic dopamine transmission with positron emission tomography. Part II: amphetamineinduced dopamine release in the functional subdivisions of the striatum. J Cereb Blood Flow Metab 23: 285-300.

McGowan S, Lawrence AD, Sales T, Quested D, Grasby P (2004). Presynaptic dopaminergic dysfunction in schizophrenia: a positron emission tomographic [18F]fluorodopa study. Arch Gen Psychiatry 61: 134-142.

Meyer JH, Gunn RN, Myers R, Grasby PM (1999). Assessment of spatial normalization of PET ligand images using ligand-specific templates. Neuroimage 9: 545-553.

Montgomery AJ, Thielemans K, Mehta MA, Turkheimer F, Mustafovic S, Grasby PM (2006). Correction of head movement on PET studies: comparison of methods. J Nucl Med 47: 1936-1944.

Morgan D, Grant KA, Gage HD, Mach RH, Kaplan JR, Prioleau O et al (2002). Social dominance in monkeys: dopamine D2 receptors and cocaine self-administration. Nat Neurosci 5: 169-174. 
Pappata S, Dehaene S, Poline JB, Gregoire MC, Jobert A, Delforge J et al (2002). In vivo detection of striatal dopamine release during reward: a PET study with [(11)C] raclopride and a single dynamic scan approach. Neuroimage 16: 1015-1027.

Patlak CS, Blasberg RG (1985). Graphical evaluation of blood-tobrain transfer constants from multiple-time uptake data. Generalizations. J Cereb Blood Flow Metab 5: 584-590.

Pinborg LH, Arfan H, Haugbol S, Kyvik KO, Hjelmborg JV, Svarer $\mathrm{C}$ et al (2008). The 5-HT2A receptor binding pattern in the human brain is strongly genetically determined. Neuroimage 40 : $1175-1180$.

Pruessner JC, Champagne F, Meaney MJ, Dagher A (2004). Dopamine release in response to a psychological stress in humans and its relationship to early life maternal care: a positron emission tomography study using [11C]raclopride. J Neurosci 24: 2825-2831.

Reed T, Plassman BL, Tanner CM, Dick DM, Rinehart SA, Nichols WC (2005). Verification of self-report of zygosity determined via DNA testing in a subset of the NAS-NRC twin registry 40 years later. Twin Res Hum Genet 8: 362-367.

Reeves SJ, Grasby PM, Howard RJ, Bantick RA, Asselin MC, Mehta MA (2005). A positron emission tomography (PET) investigation of the role of striatal dopamine (D2) receptor availability in spatial cognition. Neuroimage 28: 216-226.
Reeves SJ, Mehta MA, Montgomery AJ, Amiras D, Egerton A, Howard RJ et al (2007). Striatal dopamine (D2) receptor availability predicts socially desirable responding. Neuroimage 34: 1782-1789.

Schott BH, Minuzzi L, Krebs RM, Elmenhorst D, Lang M, Winz OH et al (2008). Mesolimbic functional magnetic resonance imaging activations during reward anticipation correlate with reward-related ventral striatal dopamine release. J Neurosci 28: 14311-14319.

Shotbolt P, Stokes PR, Owens SF, Toulopoulou T, Picchioni MM, Bose SK et al (2011). Striatal dopamine synthesis capacity in twins discordant for schizophrenia. Psychol Med 41: 2331-2338.

Volkow ND, Wang GJ, Telang F, Fowler JS, Logan J, Jayne M et al (2007). Profound decreases in dopamine release in striatum in detoxified alcoholics: possible orbitofrontal involvement. J Neurosci 27: 12700-12706.

Wahl L, Chirakal R, Firnau G, Garnett ES, Nahmias C (1994). The distribution and kinetics of [18F]6-fluoro-3-O-methyl-L-dopa in the human brain. J Cereb Blood Flow Metab 14: 664-670.

Willeit M, Ginovart N, Graff A, Rusjan P, Vitcu I, Houle S et al (2008). First human evidence of d-amphetamine induced displacement of a D2/3 agonist radioligand: A [11C]-(+)-PHNO positron emission tomography study. Neuropsychopharmacology 33: $279-289$.

Supplementary Information accompanies the paper on the Neuropsychopharmacology website (http://www.nature.com/npp) 